\title{
A Monolithically Fabricated Combinatorial Mixer for Microchip-Based High-Throughput Cell Culturing Assays
}

\author{
Mike C. Liu ${ }^{1 *}$, Dean $\mathrm{Ho}^{2}$ and Yu-Chong Tai ${ }^{1}$ \\ ${ }^{1}$ Caltech Micromachining Laboratory, California Institute of Technology, Pasadena, CA, U.S.A. \\ ${ }^{2}$ Department of Biomedical and Mechanical Engineering, Northwestern University, IL, U.S.A.
}

\begin{abstract}
We present an integrated method to fabricate 3D microfluidic networks and fabricated the first on-chip cell culture device with an integrated combinatorial mixer. The combinatorial mixer is designed for screening the combinatorial effects of different compounds on cells. The monolithic fabrication method with parylene $\mathrm{C}$ as the basic structural material allows us to avoid wafer bonding and achieves precise alignment between microfluidic channels. As a proof-of-concept, we fabricated a device with a three-input combinatorial mixer and demonstrated that the mixer can produce all the possible combinations. Also, we demonstrated the ability to culture cells on-chip and performed a simple cell assay on-chip using trypan blue to stain dead cells.
\end{abstract}

\section{Keywords - 3-D microfluidic; Cell Culture; Parylene C}

\section{INTRODUCTION}

Over the past decade, there has been an increase in demand for high-throughput cell-based assays for drug screening and system biology. Microfabrication technologies enable the realization of performing assay on chip with the advantages of high through-put screening, reduced sample volume and mass-fabrication of high-density arrays. Recently, with the advancements in microfabrication and microfluidic technologies, there have been many developments of lab-onchip devices for cell culturing [1] [2] [3]. Some on-chip system has the ability to culture the cells under the gradient of a single compound [4]. However, most devices only have the ability to screen for only one compound and there has been no cell culture device capable of assaying for the combinatorial effect of several compounds on cells. The ability of the chip to screen for the combinatorial effect of several compounds has great importance because cells receive more than one extracellular signal at once. Cells divide, survive, grow, differentiate, or undergo apoptosis as a result of the integration of the various different signals that they receive [5]. Also, biological phenomenons such as gene regulation or stem cell differentiation control are governed by the combinatorial effects of several gene regulatory proteins or extracellular factors. In addition, combination therapy has been standard in treatment of HIV, and study has also shown that the combinatorial effect of two or more drugs can improve chemotherapy [6]. A 3-D microfluidic device with a combinatorial mixer is required for on-chip screening of the combinatorial effect of compounds on cells, but previous methods of fabricating combinatorial mixers often involve patterning and bonding of different layers. For example, several layers of glass were wet etched and drilled, and then the patterned glasses layers were bonded together by thermal bonding [7]. Other materials such as PDMS or Mylar have been used with different methods of patterning, but they still require layer-to-layer bonding [8] [9]. Such methods suffer the drawbacks of imprecise alignment between channels, and the lower yield of fabrication. Therefore a monolithically fabricated 3-D microfluidic device for cell culturing is desirable. Our goal is to fabricate a device utilizing an integrated method to monolithically fabricate the 3-D microfluidic networks and the cell culture chambers.

\section{DESIGN}

Figure 1 shows the device design layout. The device has a combinatorial mixer with three inputs, and the combinatorial mixer recombines those inputs into the seven possible outputs. For example, compounds $\mathrm{A}, \mathrm{B}$, and $\mathrm{C}$, will be recombined into $\mathrm{A}, \mathrm{B}, \mathrm{C}, \mathrm{A}+\mathrm{B}, \mathrm{A}+\mathrm{C}, \mathrm{B}+\mathrm{C}$, and $\mathrm{A}+\mathrm{B}+\mathrm{C}$. One control channel that does not receive any of the three inputs is also included. The combinatorial mixer has several "overpass" structures that allows one microfluidic channel to cross over another microfluidic channel. The cell loading port is where the cells will be introduced and where ambient air can enter into the culture chamber. The cells will be grown inside the culture chamber.

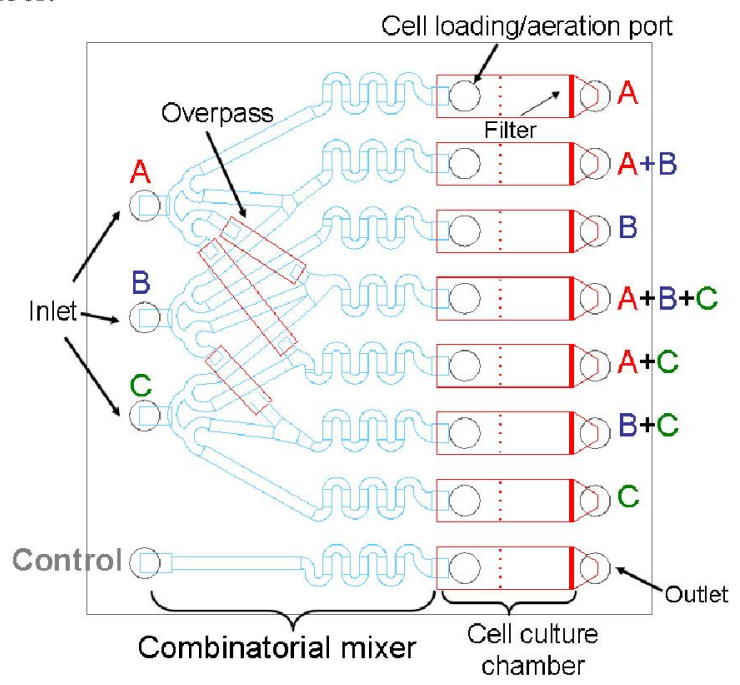

Figure 1. Design Layout of Device

\footnotetext{
* Contacting Author: Mike C. Liu is with Caltech Micromachining Lab, California Institute of Technology, 1200 E. California Blvd, Pasadena, CA 91125, USA (phone: 1-626-395-3884; email: mikeliu@caltech.edu).
} 


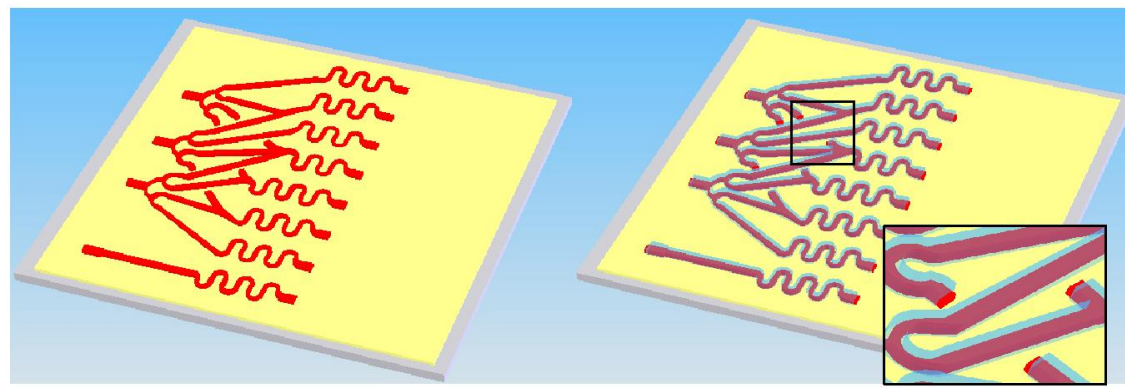

(1) Deposit $1^{\text {st }}$ layer parylene; Define $1^{\text {st }}$ sacrificial photoresist layer
(2) Deposit $2^{\text {nd }}$ layer parylene and pattern

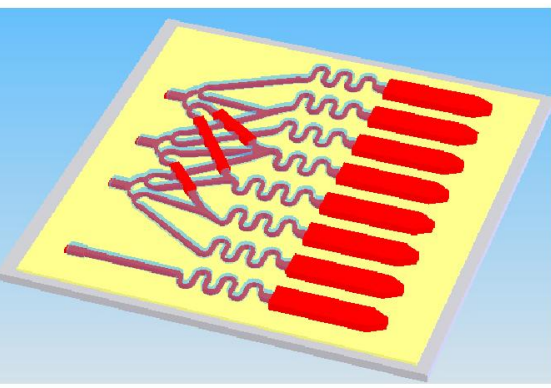

(3) Define $2^{\text {nd }}$ sacrificial photoresist layer

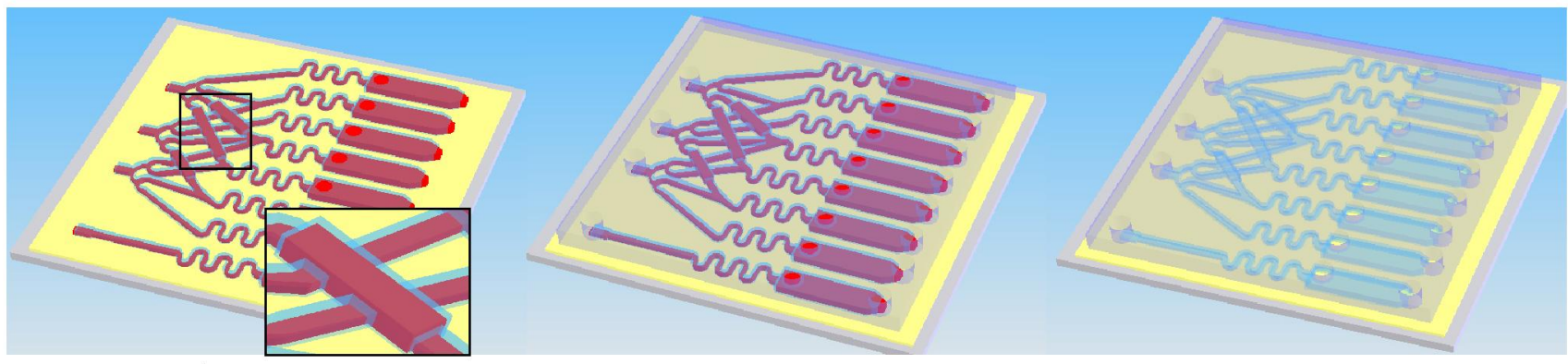

(4) Deposit $3^{\text {rd }}$ layer Parylene and pattern

(5) SU8 planarization
(6) Release in IPA

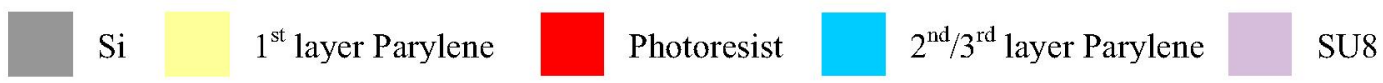

Figure 2. Fabrication steps. Inserts in (2) and (4) show how the overpass is made by first etching open the parylene and join the two etched regions with the $2^{\text {nd }}$ sacrificial photoresist.

The device is realized by the surface micromaching of parylene $\mathrm{C}$. Parylene $\mathrm{C}$ is compatible with lithographic CMOS/MEMS fabrication and can be processed using microfabrication techniques such as reactive ion etching [10]. This allows the device to be monolithically fabricated, and such method yields 3-D microfluidic devices with precise alignment between channels. Also, Parylene $\mathrm{C}$ has proved to be chemically inert, as it has been used in many implantable devices [11]. Chemically inertness is an important property because any byproduct generated from the breakdown of the culture chamber structures can deleteriously affect the cell culture experiments. In addition, Parylene $\mathrm{C}$ is transparent in the visible range and will allow the cells to be easily observed with light microscopy. Therefore, Parylene $\mathrm{C}$ is the ideal material for the monolithic fabrication of cell culture chip with an integrated combinatorial mixer.

\section{FABRICATION}

The device was fabricated using a simple 5-mask process and the fabrication steps are shown in figure 2. First, silicon wafer was cleaned for processing. The first layer of Parylene $\mathrm{C}$ was deposited $(3 \mu \mathrm{m})$ with a Cookson Electronics ${ }^{\mathrm{TM}}$ PDS 2010 system (Specialty Coating Systems Inc., Indianapolis, IN). The first sacrificial photoresist layer (AZ4620 from Clariant Corp., Charlotte, NC) was spin-coated $(15 \mu \mathrm{m})$ and patterned to define the first-layer channels. The sacrificial photoresist was hard-baked for degassing purposes. Then, a second Parylene $\mathrm{C}$ layer was deposited to form the first-layer channels. Parylene $\mathrm{C}$ was then patterned using oxygen plasma so the areas where the overpass structure would be joined were exposed. This Parylene patterning also opened the area where the mixer and the culture chamber would be connected. A second sacrificial photoresist was spin-coated $(35 \mu \mathrm{m})$ to define the overpass structure and also the culture chamber. A third layer Parylene $C(10 \mu \mathrm{m})$ was deposited and patterned to define the access holes. The whole device was planarized with thick SU8 $(100 \mu \mathrm{m})$ to facilitate future packaging of the device. The wafer was diced to yield devices with dimensions of $1 \mathrm{~cm}$ by $1 \mathrm{~cm}$. Finally, the devices were soaked in IPA to elute out the sacrificial photoresist. Our fabrication method allows tremendous ability for scaling up the number of inputs. By using the method of first laying down the basic channels and then making the overpasses, we will be able to make device with more inputs using the same number of steps.

\section{A. Device Fabrication}

\section{RESULTS/DISCUSSION}

The fabricated device is shown in figure 3. Figure 4 and figure 5 show the successful fabrication of microfluidic overpass structures. SEM image (Figure 4) of the crosssection of the overpass structure shows that the two fluid streams will be separated spatially at the overpass region. Figure 5 shows the surface profile scan (P-15 stylus profilometer from KLA-Tencor, San Jose, Ca) of the overpass 
structure before SU8 planarization and it shows that the overpass structure is about 15 to $20 \mu \mathrm{m}$ higher than the $1^{\text {st }}$ layer microfluidic channels.

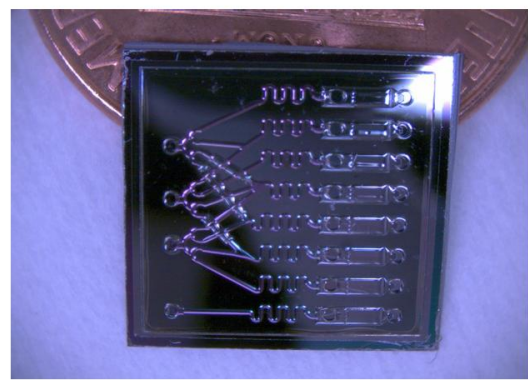

Figure 3. Fabricated device, $1 \mathrm{~cm}$ by $1 \mathrm{~cm}$

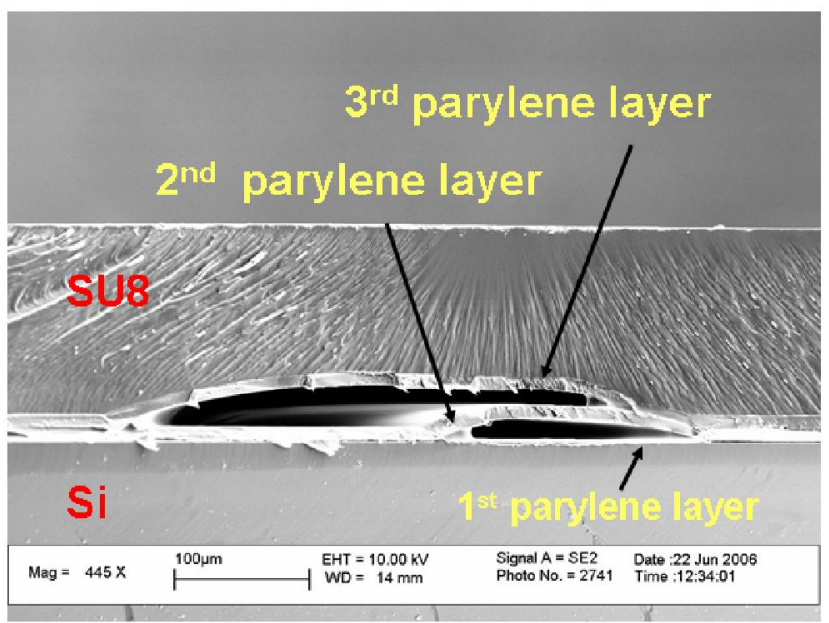

Figure 4. SEM image of the cross-sectional view of the microfluidic overpass

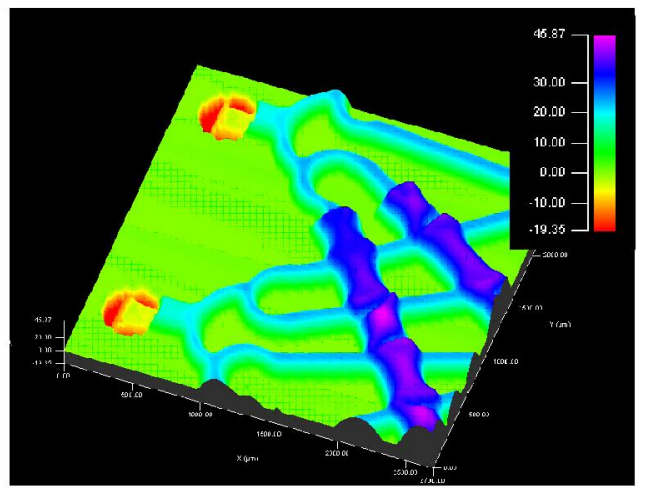

Figure 5. Surface profile scan of the microfluidic overpass

\section{B. Device Packaging and Operation}

The device is packaged in two different ways for cell loading or combinatorial assays (Figure 6). The packaging is done by aligning a piece of PDMS with punched holes on the chip and sandwich them with two pieces of acrylics. Teflon tubes are plugged into the PDMS for fluidic connections. Figure 7 shows the schematic of how the device will operate. First the device will be packaged for cell loading, as shown in
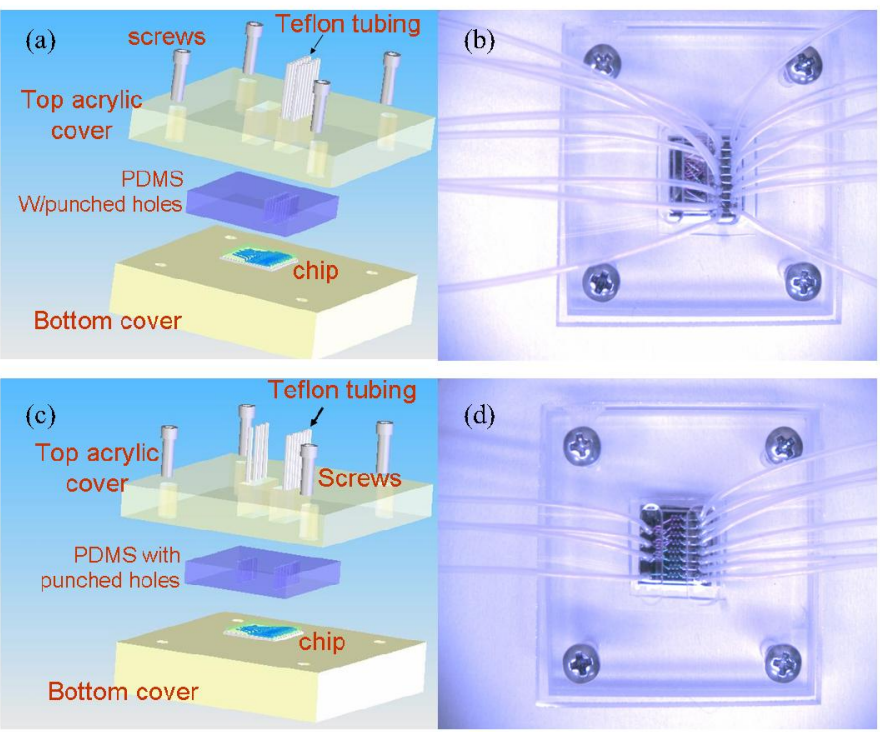

Figure 6. Device Packaging: (a) 3-D rendition of packaging for cell loading. (b) Picture of device packaged for cell loading. (c) 3-D rendition of packaging for combinatorial assaying. (d) Picture of device packaged for combinatorial assaying

(a)

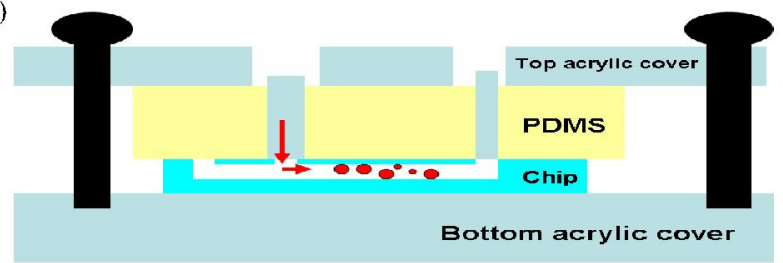

(b)

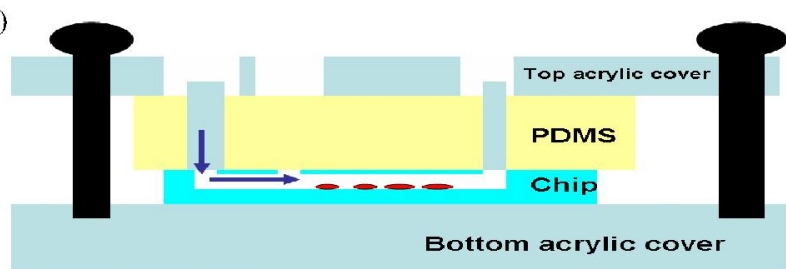

Figure 7. Device Operation: (a) Cell loading and adhering. (b) After cells attach, reassemble the packaging with a different piece of PDMS for perfusion with the combinatorial mixer

figure $6 \mathrm{a}$ and $6 \mathrm{~b}$. The cells will be introduced into the culture chambers with a syringe. After two to four hours, the cell will adhere to the surface. Then, the device will be packaged for combinatorial assaying. Figure $6 \mathrm{c}$ and $6 \mathrm{~d}$ show the device packaged for combinatorial assaying. The setups for cell loading and combinatorial assays are almost the same, except the PDMS pieces have access holes at different places.

\section{Combinatorial Mixer Testing}

Figure 8 shows the testing of the combinatorial mixer. Green, red and blue food colorings are injected into the three input ports by syringe pumps, while yellow is used for the control. The images were taken with a stereoscope equipped with a CCD camera. The result shows that we have successfully constructed the combinatorial mixer, as figure 8 shows that combinatorial mixer is able to recombine the three inputs into the seven possible combinations. Figure 9 shows 
mixing results at two different flow rates, and as flow rate goes down, molecules have more time to diffuse across the channel for better mixing.

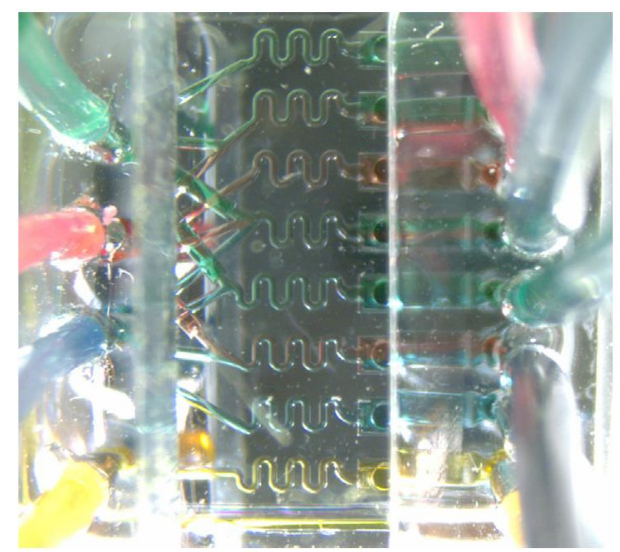

Figure 8. Combinatorial mixer: all possible combinations from green, red and blue food colorings are generated. Flow rate at $100 \mu \mathrm{L} / \mathrm{min}$

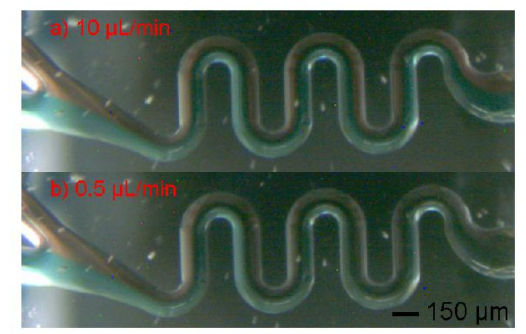

Figure 9. Mixing inside the serpentine channel at different flow rates.

\section{Cell Culturing}

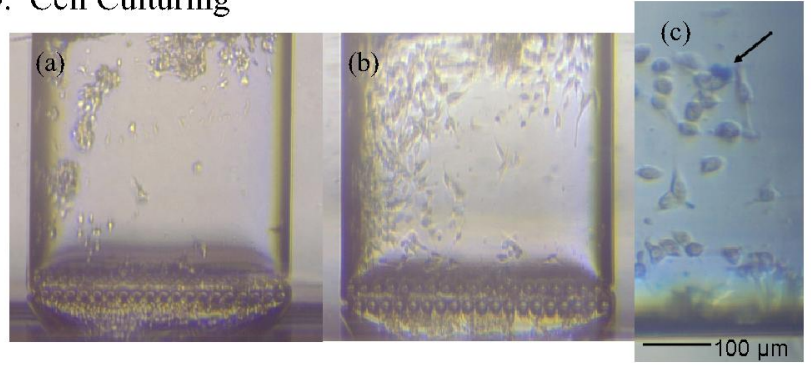

Figure 10. Cell culturing: (a) Cells loaded and attached; (b) Cells spread, proliferated after 16 hours of perfusion of culture media; (c) Cell assay with trypan blue stain: live cells exclude trypan blue, while dead cells are stained blue (arrow).

The chip was sterilized by UV irradiation and treating all the fluidic channels and culture chambers with $70 \%$ ethanol for 30 minutes. To culture the cells, we first coated the chamber with $0.05 \%$ Polyethyleneimine (PEI) in borate buffer solution to enhance cell adhesion. B35 rat neuroblastoma cells are injected into the culture chambers using a syringe and adhered to the chamber surface in four hours (Figure 9a). Then, cells are cultured with continuous perfusion of culture media (Dulbecco's Modified Eagle's Medium with 10\% Fetal Bovine Serum, 1\% Penicillin/Streptomycin/Amphotericin B) at flow rate of $33 \mathrm{~nL} / \mathrm{min}$, which corresponds to turning over the entire culture chamber per minute. The device is kept inside a $37^{\circ} \mathrm{C}$ incubator with $5 \% \mathrm{CO}_{2}$. Figure $9 \mathrm{~b}$ shows that after 16 hours of perfusion, cells have spread and proliferated. To demonstrate the ability to perform on chip cell assay, we loaded 2\% trypan blue solution to stain dead cells (Figure 9c).

\section{CONCLUSION}

We have successfully fabricated the chip for cell culturing with an integrated combinatorial mixer. Our fabrication method is relatively simple as only five masks are required and this method can also be easily scaled up to include more inputs for the combinatorial mixer. Our monolithic fabrication method allows us to avoid the laborious bonding step and achieve precise alignment between channels. Also, we developed a simple and inexpensive method for packaging the device and making the fluidic connections. The fluidic testing shows that the microfluidic overpass structure was fabricated successfully and all the right combinations from three input streams were generated. We have used this chip to culture cells and demonstrated cell assay on chip. In the future, cell culturing under combinatorial conditions will be performed with this device.

\section{REFERENCES}

[1] A. Prokop, Z. Prokop, D. Schaffer, E. Kozlov, J. Wikswo, D. Cliffel, and F. Baudenbacher, "NanoLiterBioReactor: Long-term mammalian cell culture at nanofabricated scale," Biomedical Microdevices, vol 6, pp. 325-339, 2004.

[2] P.J. Hung, P.J. Lee, P. Sabounchi, R. Lin, and L.P. Lee, "Continuous perfusion microfluidic cell culture array for high-throughput cell-based assays," Biotechnology and Bioengineering, vol 89, pp. 1-8, 2005.

[3] V. I. Chin, P. Taupin, S. Sanga, J. Scheel, F. H. Gage and S. N. Bhatia "Microfabricated platform for studying stem cell fates." Biotechnology and Bioengineering, vol 88, pp. 401-415, 2004.

[4] P. J. Lee, P. J. Hung, V. M. Rao, and L. P. Lee, "Nanoliter scale microbioreactor array for quantitative cell biology." Biotechnology and Bioengineering, vol 94, pp. 5-14, 2005.

[5] A. Bruce et al., Molecular Biology of the Cell, 4th ed., New York, NY: Garland Science, 2002, pp. 831-906.

[6] H. G. Wendel1, E. de Stanchina, J. S. Fridman1, A. Malina, S. Ray, S. Kogan, C. Cordon-Cardo, J. Pelletier, and S. W. Lowe1, "Survival signalling by Akt and eIF4E in oncogenesis and cancerr therapy," Nature, vol 428, pp. 332-337, 2004.

[7] Y. Kikutani, T. Horiuchi, K. Uchiyama, H. Hisamoto, M. Tokeshia, and T. Kitamori, "Glass microchip with three-dimensional microchannel network for 2 X 2 parallel synthesis." Lab on a Chip, vol 2, pp. 188192, 2002.

[8] R. F. Ismagilov, J. M. K. Ng, P. J. A. Kenis, and G. M. Whitesides, "Microfluidic arrays of fluid-fluid diffusional contacts as detection elements and combinatorial tools," Anal. Chem., vol 73, pp. 5207-5213, 2001 .

[9] C. Neils, Z. Tyree, B. Finlayson, and A. Folch, "Combinatorial mixing of microfluidic streams," Lab on a Chip, vol. 4, pp. 342-350, 2004.

[10] J. T. C. Yeh and K. R. Grebe, "Patterning of poly-para-xylylenes by reactive ion etching," Journal of Vacuum Science \& Technology aVacuum Surfaces and Films, vol.1, pp. 604-608, 1983.

[11] B. D Ratner et al, An Introduction to Materials in Medicine, 1st ed., London, UK: Academic Press, 1996, pp. 115-116. 